Itinéraires Itinéraires

Littérature, textes, cultures

2009-2 | 2009

Caraïbe et océan Indien

\title{
Kumari R. Issur et Vinesh Y. Hookoomsing (dir.), L'océan Indien dans les littératures francophones
}

\section{Alexandra Bourse}

\section{(2) OpenEdition}

\section{Journals}

Édition électronique

URL : http://journals.openedition.org/itineraires/209

DOI : 10.4000/itineraires.209

ISSN : 2427-920X

Éditeur

Pléiade

\section{Édition imprimée}

Date de publication : 1 juillet 2009

Pagination : 167-171

ISBN : 978-2-296-09639-4

ISSN : $2100-1340$

\section{Référence électronique}

Alexandra Bourse, « Kumari R. Issur et Vinesh Y. Hookoomsing (dir.), L'océan Indien dans les littératures francophones », Itinéraires [En ligne], 2009-2 | 2009, mis en ligne le 23 mai 2014, consulté le 22

septembre 2020. URL : http://journals.openedition.org/itineraires/209 ; DOI : https://doi.org/10.4000/ itineraires.209

Ce document a été généré automatiquement le 22 septembre 2020.

\section{cc) $(1) \odot$}

Itinéraires est mis à disposition selon les termes de la licence Creative Commons Attribution - Pas d'Utilisation Commerciale - Pas de Modification 4.0 International. 


\title{
Kumari R. Issur et Vinesh Y. Hookoomsing (dir.), L'océan Indien dans les littératures francophones
}

\author{
Alexandra Bourse
}

\section{RÉFÉRENCE}

Kumari R. Issur et Vinesh Y. Hookoomsing (dir.), L'océan Indien dans les littératures francophones, Karthala - Presses de l'Université de Maurice, 2001, 706 pages, ISBN : 2-84586-225-3.

1 Cet ouvrage collectif présente les actes d'un colloque organisé par l'université de Maurice en juillet 1997. Il vise à ouvrir la littérature insulaire de l'océan Indien à « un espace francophone régional élargi » (4). Les littératures de l'océan Indien, rappellent les directeurs du colloque, comprennent en effet les productions de l'île Maurice, de La Réunion, de Madagascar mais aussi celles issues des relations entretenues avec l'Inde, la France et l'Angleterre.

2 Ces actes évoquent à la fois la vision occidentale des îles - exotisme que les intervenants analysent grâce à la critique énoncée par Edward Said dans Orientalism - et la difficulté à rendre compte de la particularité d'imaginaires et de langues forgés dans le contexte de la double colonisation française et anglaise. Les propos élaborent ainsi des liens entre des visions occidentales fantasmées de ces îles et des stratégies à la fois linguistiques, discursives et poétiques afin de les remettre en question, voire de les écarter totalement.

3 La première partie de l'ouvrage, intitulée "Voyages et rencontres ", propose la généalogie des relations entre les îles de l'océan Indien et l'Occident. Margaret Sankey, dans son article « Est et Ouest : le mythe des terres australes en France aux XVII ${ }^{\mathrm{e}}$ et XVIII siècles ", souligne que l'apparition d'une littérature utopique à cette époque a favorisé l'exploration de ces îles et s'est associée à d'autres intérêts, économiques en particulier 
(s'assurer des postes d'établissement sur la route des Indes) mais aussi religieux (convertir les âmes). La vogue des récits de voyages, réels ou fictifs, dans la seconde moitié du XVII siècle fut donc simultanément un moteur et un réceptacle pour ces explorations. À cette littérature s'ajoutent bien plus tard, dans la mise en place d'une image fantasmée des îles de l'océan Indien, les expositions coloniales organisées en France entre le milieu du XIX siècle et la fin des années 1930. Si un discours de reconnaissance du métissage culturel affleure dans ces textes et ces expositions, il n'en reste pas moins que prédominent, jusqu'au $\mathrm{xx}^{\mathrm{e}}$ siècle, stéréotypes et préjugés allant d'une idéalisation aveugle de l'insularité (notamment liée à une nostalgie du paradis perdu) à des jugements racistes où transparaît une hiérarchisation des races, ce que montre Gérard Fanchin dans «L'anthropologie des Lumières et ses résonances dans les relations de voyage à l'Isle de France aux XVIII ${ }^{\mathrm{e}}$ et XIX ${ }^{\mathrm{e}}$ siècles ».

4 La deuxième partie de l'ouvrage, intitulée «Mer indienne: Crédité et Indianocéanisme ", est sans doute la plus intéressante pour comprendre les littératures insulaires contemporaines, notamment grâce à une notion conceptualisée par Camille de Rauville à la fin des années 1960. En effet, comme le précise Michel Beniamino dans "Camille de Rauville et l'indianocéanisme ", il a fallu attendre les années 1950-1960 pour que soit repensée cette aire géographique avec la constitution de diverses associations analysant les bouleversements nés de la fin de la présence anglaise, des convoitises américaine, russe mais aussi chinoise. Dans ce contexte, un réel désir d'autonomie s'est fait sentir et a supposé l'invention de nouvelles modalités discursives susceptibles d'en rendre compte. La démarche comparatiste de Rauville prend en compte les diversités et les convergences culturelles de cet espace, le métissage des langues et des religions. Ce dernier conçoit, à partir de cette hétérogénéité, un ensemble cohérent fondé sur les rapports entretenus entre les différentes îles. L'apport hindou, le fantasme du métissage et la mise en place de nouvelles stratégies politiques et culturelles lors de la période post-coloniale sont considérés comme des critères homogénéisants. Cependant, cette volonté d'homogénéisation, prégnante dans le néologisme "indianocéanisme ", pousse à nier les particularités, dévalorisant notamment les créoles au profit des langues des anciens dominants (anglais et français); coup de force assez dangereux susceptible d'aboutir à l'effacement de cultures jugées subalternes, qui seraient hostiles à une véritable cohésion. Ce concept est entré en relation et en contradiction avec celui de "créolie » fondé par le poète réunionnais Jean Albany. Mais la créolie insiste sur le métissage biologique et culturel, si bien que, pour Camille de Rauville, elle reflète une sensibilité nationale (réunionnaise) à laquelle les Mascareignes ne peuvent s'identifier du fait de leur caractère multiracial et plurilingue. Rauville insiste davantage sur un "métissage psychique ${ }^{1}$ ", coup de force idéologique prenant en compte les "contraintes sociosymboliques » (98) propres à cette aire géographique. Les deux concepts proposent bien deux visions différentes du métissage. L'indianocéanisme cherche en réalité à se présenter comme un nouvel humanisme doté d'une prétention à l'universalité, ce que l'article de Jean-Georges Prosper, «Le créole indianocéaniste: un humanisme planétaire ", montre bien. Il propose en effet une vision extrêmement idéalisée et univoque d'une « culture globale humaniste » (109) unifiante, au risque d'une exclusion des particularités et des individualités.

5 Les articles suivants interrogent l'émergence de la littérature dans les différentes îles de l'océan Indien. Les écrivains cherchent souvent une publication en Occident, seule 
apte aujourd'hui à leur conférer une véritable visibilité. Une politique éditoriale semble nécessaire pour aider ces littératures à sortir de l'ombre.

6 La troisième partie de l'ouvrage centre son propos sur une analyse pluridisciplinaire des imaginaires malgaches: approche de la poésie d'abord, avec l'article de Liliane Ramarosoa sur «les imaginaires de l'île à travers la poésie malgache d'expression française » qui revient sur le parcours de l'écrivain J.-J. Rabearivelo, lequel partage avec Jacques Rabemananjara une nostalgie du passé et une vision pessimiste de l'histoire, format manichéen détruit après 1975 par une nouvelle génération de poètes mettant l'histoire entre parenthèses pour s'attacher à la peinture du présent, entre violence et moments de bonheur intenses. Suzanne Chazan-Gillig propose quant à elle, dans son article, une confrontation entre analyse anthropologique et écriture romanesque autour de l'expérience du "Bain des reliques" à Madagascar, expérience de la transgression partagée avec Michèle Rakotoson, laquelle a publié un roman sur cette cérémonie en 1988. Michèle R. Ratovonony-Ratsirahonana analyse ainsi « les figures de rêves" (223) qui se manifestent dans cet ouvrage intitulé précisément Le Bain des reliques.

7 Les quatrième et cinquième parties de L'océan Indien dans les littératures francophones traitent de «l'interculturalité » d'un point de vue littéraire et linguistique. Elles sont le lieu d'analyses des théories particularistes, tentatives de mise en valeur d'identités refusant de n'être que des minorités dans ces espaces multiculturels. La prégnance du français par exemple suggère la mise en valeur d'autres cultures avec une insistance particulière sur le versant linguistique. Vinesh Y.Hookoomsing traite ainsi de la " coolitude ", concept imaginé par Khal Torabully pour exprimer l'identité propre aux Indiens qui ont vécu l'expérience de l'exil et de l'engagisme. Le problème de cette notion, selon l'auteur de l'article, vient de ce que cette démarche « consiste à vouloir à tout prix calquer le rapport entre coolitude et indianité sur la relation bien établie entre négritude et créolité » (257). Or, le déracinement subi par l'immigrant indien n'est pas le même déracinement que celui de l'esclave africain ; par ailleurs, les Indiens ont mieux réussi à se réapproprier l'espace de l'exil, à y perpétuer leurs traditions culturelles. De la même manière, les Tamouls ont tenté de définir leur identité en développant une presse tamoule relayant leurs combats d'ordre ethnique, religieux et politique. Les articles mettent en lumière les différentes revendications identitaires, les luttes qu'elles engendrent, les divisions et les échecs propres à toute société multiculturelle. Affronter la diversité est de la même manière l'objet principal des œuvres littéraires de cet espace géographique, ce que Marcel Cabon par exemple, dans son roman Namasté, met en évidence sur un mode tragique grâce à son protagoniste, Ram, sacrifié au nom du rejet de l'altérité. Carl de Souza, de son côté, problématise les rapports conflictuels entre les différentes communautés mauriciennes qui se rencontrent et s'opposent, ce qu'analyse Cécile Leung dans «Le modèle ambigu de l'interaction sociale au sein du microcosme mauricien dans La Maison qui marchait vers le large de Carl de Souza ».

8 Un des aspects du colloque s'intéresse également à un autre mode d'exclusion aujourd'hui battu en brèche et qui relève cette fois du genre (au sens du gender anglosaxon). Dans les littératures de l'océan Indien, ce sont d'abord des auteurs masculins qui ont obtenu une reconnaissance. Les femmes restaient dans l'ombre. L'article de Jeanne Gerval-Arouff, "Femme et territoire : état de la femme écrivain», souligne l'émergence de la parole féminine cherchant à être préservée de «l'immémoire ». Les 
premiers textes de femmes ont d'abord été des revendications souvent anonymes à caractère social ou des textes féministes, à l'image de ceux de Rada Gungaloo. On remarque dans les romans de femmes la difficulté de trouver une place dans l'espace de l'île (Nathacha Pathareddy-Appanah), le rêve d'une communauté soudée, la mise en valeur des rapports mère-enfants (Shenaz Patel) ou bien encore la dénonciation de la misère et de ses horreurs (Ananda Devi). Ces écritures cherchent à briser, entre autres, les stéréotypes liés à la perception phallocentrique de la féminité, perception que JeanClaude Carpanin Marimoutou étudie à travers l'analyse du fantasme de «La Belle Créole » à La Réunion.

9 Les septième et huitième parties de l'ouvrage s'intéressent respectivement à la relation qu'entretiennent ces îles (mais aussi la France) avec l'Inde: espace à la fois rêvé (en témoigne le titre de l'article de Radha Sharma, "Rêver l'Inde à travers la littérature française »), interrogé et dévalorisé (notamment dans les années 1960-1970 où toute une littérature véhicule l'image d'une Inde plongée dans la misère, la stagnation, insistant sur le personnage du Paria et de l'Intouchable) et, dans une perspective plus large, à l'analyse des relations entre l'Afrique, les Caraïbes et les Mascareignes. Plusieurs articles évoquent les œuvres de Le Clézio. Ils insistent sur sa déconstruction des différents stéréotypes et sa vision plus juste de l'Autre, montrant notamment comment il revisite, notamment dans Le Chercheur d'or, sur un mode critique, Paul et Virginie de Bernardin de Saint-Pierre, mais aussi le mythe de Robinson (comme Michel Tournier dans Vendredi ou les limbes du Pacifique). Concernant les relations entre l'Afrique, les Caraïbes et les Mascareignes, l'article de Joyce Leung, « Épistèmès : l'étude du savoir dans les littératures de la canneraie ", analyse les convergences entre les modes d'être et de savoir de personnages en lutte pour la liberté pendant et après la période esclavagiste, à la Martinique, en Guadeloupe, à La Réunion et à l'île Maurice.

Ce colloque a permis, ainsi que le précisent Kumari R. Issur et Vinesh Y. Hookoomsing, la création de l'Association internationale d'études francophones et comparées sur l'océan Indien, en décembre 1998. La réflexion a eu un réel impact sur la reconnaissance d'un espace littéraire dont la multiculturalité, perçue comme un ensemble hétérogène difficile à appréhender, fut longtemps considérée comme un obstacle à son identification comme champ littéraire.

\section{NOTES}

1. Camille de Rauville, Littératures francophones de l'océan Indien, Saint-Denis (La Réunion), Éditions du Tramail, 1990, p. 34. 


\section{AUTEURS}

ALEXANDRA BOURSE

Université Paris 13, CENEL 